\title{
A supnorm estimate for one-dimensional porous medium equations with advection
}

\section{Juliana S. Ziebell ${ }^{1}$}

Instituto de Matemática, Estatística e Física, FURG, Santo Antônio da Patrulha, RS

Lucinéia Fabris ${ }^{2}$

Coordenadoria Acadêmica, UFSM, Cachoeira do Sul, RS

Janaina P. Zingano ${ }^{3}$

Instituto de Matemática, UFRGS, Porto Alegre, RS

Linéia Schütz ${ }^{4}$

Instituto de Matemática, UFRGS, Porto Alegre, RS

Abstract. We give a short derivation of supnorm estimates for solutions of one-dimensional porous medium equations of the form

$$
u_{t}+(f(t, u))_{x}=\left(|u|^{\alpha} u_{x}\right)_{x},
$$

assuming initial data $u(\cdot, 0) \in L^{p_{0}}(\mathbb{R}) \cap L^{\infty}(\mathbb{R})$ for some $1 \leq p_{0}<\infty$.

Key-words. Porous Medium Equation, Supnorm Estimate, Comparison Theorem

\section{Introduction}

There are a number of physical applications where the porous medium equation describes processes involving fluid flow, heat transfer or diffusion [5]. The porous medium equation without advection is given by

$$
\frac{\partial u}{\partial t}=\left(|u|^{m-1} u\right)_{x x}+f, m>1,
$$

where $f=f(x, t)$ is a source term.

Here we consider the following initial-value problem

$$
\left\{\begin{array}{l}
u_{t}+(f(t, u))_{x}=\left(|u|^{\alpha} u_{x}\right)_{x}, x \in \mathbb{R}, t>0, \\
u(\cdot, 0)=u_{0} \in L^{p_{0}}(\mathbb{R}) \cap L^{\infty}(\mathbb{R}), 1 \leq p_{0}<\infty,
\end{array}\right.
$$

\footnotetext{
${ }^{1}$ julianaziebell@furg.br

${ }^{2}$ lucineia.fabris@ufsm.br

3 jzingano@mat.ufrgs.br

${ }^{4}$ lineia.schutz@ufrgs.br
} 
where $\alpha \geq 0$ and $f \in C^{1}([0, \infty) \times \mathbb{R})$ are given. The solutions of (2) are known to be defined for all $t>0$ and decay as $t \rightarrow \infty$ in several spaces. In this work, we derive a supnorm estimate for the solutions of $(2)$ when considering $u(\cdot, 0)$ in $L^{p}(\mathbb{R}), p=p_{0}+\alpha / 2$. By solution in some interval $\left[0, T^{*}\right), 0<T^{*} \leq \infty$, we mean a measurable function $u: \mathbb{R} \times\left[0, T^{*}\right) \rightarrow \mathbb{R}$ which is bounded in each strip $\mathbb{R} \times[0, T], 0<T<T^{*}$, and which solves the equation (2) in distributional sense.

\section{Preliminary}

An important result to obtain a supnorm estimate also for negative solutions is the following Theorem.

Theorem 2.1. (Theorem of comparison:)

Let $u(\cdot, t), v(\cdot, t)$ solutions of the equation (1), with initial value $u_{0}, v_{0} \in L^{\infty}(\mathbb{R})$, respectively, both defined for $0<t<T$ and limited in the strip $\mathbb{R} \times[0, T]$. Also, if

$$
|f(x, t, u)-f(x, t, v)| \leq K_{f}(M, T)|u-v|, \forall x \in \mathbb{R}, \forall t, 0 \leq t \leq T,
$$

then

$$
u_{0}(x) \leq v_{0}(x) \text { a.e. } x \in \mathbb{R} \Rightarrow u(x, t) \leq v(x, t) \forall x \in \mathbb{R},
$$

for all $t, 0<t \leq T$.

The proof of this Theorem is in [2].

\subsection{Some importants inequalities}

The following inequalities will be important througout this work.

- For any $p, q$ and $r$ such that $0<p \leq r \leq \infty, 1 \leq q \leq \infty$ :

$$
\|w\|_{L^{r}(\mathbb{R})} \leq \tilde{K}(r, q, p)\|w\|_{L^{p}(\mathbb{R})}^{1-\tilde{\theta}}\left\|w_{x}\right\|_{L^{q}(\mathbb{R})}^{\tilde{\tilde{R}}} \forall w \in C_{0}^{1}(\mathbb{R}),
$$

where $\tilde{\theta}=\frac{1-p / r}{1+p \cdot(1-1 / q)}, \tilde{K}(r, q, p)=(2 \theta)^{-\tilde{\theta}}$ and $\theta=\frac{1}{1+p \cdot(1-1 / q)}$.

- $\forall \beta_{0}>0$ :

$$
\|u\|_{L^{\infty}(\mathbb{R})} \leq\left(\frac{2+\beta_{0}}{4}\right)\|u\|_{L^{\beta_{0}(\mathbb{R})}}^{1-\theta}\left\|u_{x}\right\|_{L^{2}(\mathbb{R})}^{\theta},
$$

where $\theta=\frac{1}{1+\frac{\beta_{0}}{2}}$. 


\subsection{Basic Result}

Theorem 2.2. If $u(\cdot, t) \in L_{\text {loc }}^{\infty}\left(\left[0, T^{*}\right), L^{\infty}(\mathbb{R})\right)$ solves problem (2) then

1) $u(\cdot, t) \in L^{p_{0}}(\mathbb{R}) \cap L^{\infty}(\mathbb{R}) \forall t, 0<t<T^{*}$

2) $\|u(\cdot, t)\|_{L^{q}(\mathbb{R})} \leq\left\|u_{0}\right\|_{L^{q}(\mathbb{R})} \forall t, \quad 0<t<T^{*}\left(\forall q, p_{0} \leq q \leq \infty\right)$

3) $\|u(\cdot, t)\|_{L^{q}(\mathbb{R})} \leq\left\|u\left(\cdot, t_{0}\right)\right\|_{L^{q}(\mathbb{R})} \forall t, 0 \leq t_{0}<t<T^{*}, \forall q, p_{0} \leq q \leq \infty$.

Proof of (1). For simplicity, we will consider the case of positive solutions, which are known to be smooth. Let $\zeta \in C^{2}(\mathbb{R})$ be such that $\zeta(x)=1 \forall|x| \leq 1, \zeta(x)=0 \forall|x| \geq 2$, $0 \leq \zeta(x) \leq 1 \forall x \in \mathbb{R}$. Given $R>0$, let $\zeta_{R}$ be the cut-off function given by $\zeta_{R}(x)=\zeta\left(\frac{x}{R}\right)$.

Let $p_{0} \leq q<\infty$. Multipliyng the PDE at the initial value problem (2) by $q u^{q-1} \zeta_{R}(x)$ we have

$$
\frac{\partial}{\partial t} u^{q} \zeta_{R}(x)+f(t, u)_{x} q u^{q-1} \zeta_{R}(x)=q u^{q-1}\left(u^{\alpha} u_{x}\right)_{x} \zeta_{R}(x) .
$$

Integrating on $\mathbb{R} \times[0, t]$,

$$
\begin{aligned}
& \int_{|x|<2 R} u(x, t) \zeta_{R}(x) d x+q(q-1) \int_{0}^{t} \int_{|x|<2 R} u^{q+\alpha-2} u_{x}^{2} \zeta_{R}(x) d x d \tau=\int_{|x|<2 R} u_{0}(x)^{q} \zeta_{R}(x) d x \\
+ & \frac{q}{q+\alpha} \int_{0}^{t} \int_{|x|<2 R} u^{q+\alpha} \zeta_{R}^{\prime \prime}(x) d x d \tau-\int_{0}^{t} \int_{|x|<2 R} f(t, u)_{x} q u^{q-1} \zeta_{R}(x) d x d \tau .
\end{aligned}
$$

Next, integrating by parts and then, letting $R \rightarrow \infty$, we get the result.

Proof of (2) and (3). Again, we consider the simpler case of positive solutions. Defining $F(t, U)=\int_{0}^{U} f^{\prime}(t, v) v^{q-1} d v$, then equation (4) can be written as

$$
\begin{aligned}
& \int_{|x|<2 R} u(x, t) \zeta_{R}(x) d x+q(q-1) \int_{0}^{t} \int_{|x|<2 R} u^{q+\alpha-2} u_{x}^{2} \zeta_{R}(x) d x d \tau=\int_{|x|<2 R} u_{0}(x)^{q} \zeta_{R}(x) d x \\
+ & \frac{q}{q+\alpha} \int_{0}^{t} \int_{|x|<2 R} u^{q+\alpha} \zeta_{R}^{\prime \prime}(x) d x d \tau+q \int_{0}^{t} \int_{|x|<2 R} F(t, u) \zeta_{R}^{\prime}(x) d x d \tau .
\end{aligned}
$$

Observe that

$$
\begin{aligned}
\int_{0}^{t} \int_{R<|x|<2 R} F(t, u) \zeta_{R}^{\prime}(x) d x d \tau & \leq \int_{0}^{t} \int_{R<|x|<2 R}|F(t, u)|\left|\zeta_{R}^{\prime}(x)\right| d x d \tau \\
& \leq \frac{M}{R} \int_{0}^{t} \int_{R<|x|<2 R}|u(x, \tau)|^{q} d x d \tau \rightarrow 0,
\end{aligned}
$$

when $R \rightarrow \infty$, where $M$ is a constant. Then

$$
\begin{aligned}
\int_{\mathbb{R}} u(x, t) d x & \leq \int_{\mathbb{R}} u(x, t)^{q} d x+q(q-1) \int_{0}^{t} \int_{\mathbb{R}} u(x, \tau)^{q+\alpha-2} u_{x}^{2} d x d \tau \\
& \leq \int_{\mathbb{R}} u_{0}(x)^{q} d x .
\end{aligned}
$$


Therefore, we get

$$
\|u(\cdot, t)\|_{L^{q}(\mathbb{R})} \leq\left\|u_{0}\right\|_{L^{q}(\mathbb{R})} \forall q, p_{0} \leq q<\infty, \forall t, 0<t<T^{*},
$$

and

$$
\|u(\cdot, t)\|_{L^{\infty}(\mathbb{R})} \leq\left\|u_{0}\right\|_{L^{\infty}(\mathbb{R})} \forall t, 0<t<T^{*} .
$$

as claimed. In particular, solutions of the initial-value problem (2) are globally defined (i.e., $T^{*}=\infty$ ).

\section{Main Theorems}

Theorem 3.1. If $u(\cdot, t) \in L_{l o c}^{\infty}\left([0, \infty), L^{\infty}(\mathbb{R})\right)$ solves problem (2) with $u_{0}>0$, then

$$
\|u(\cdot, t)\|_{L^{\infty}(\mathbb{R})} \leq K\left(\alpha, p_{0}\right)\left\|u\left(\cdot, t_{0}\right)\right\|_{L^{p_{0}(\mathbb{R})}}^{\frac{2 p_{0}}{\alpha+2 p_{0}}}\left(t-t_{0}\right)^{-\frac{1}{\alpha+2 p_{0}}}, \quad \forall t, 0 \leq t_{0}<t,
$$

where $K\left(\alpha, p_{0}\right)$ is a constant that only depends on $\alpha$ and $p_{0}$.

Proof. Let $\psi \in C^{1}(\mathbb{R})$ be monotonically increasing such that $\psi(u)=1 \forall u \geq 1, \psi(0)=0$ and $\psi(u)=-1, \forall u \leq-1$. Taking $\delta>0$, let us define $\psi_{\delta}(u)=\psi\left(\frac{u}{\delta}\right)$ and $\phi_{\delta}(u)=L_{\delta}(u)^{q}$, $q \geq 2$, where $L_{\delta}(u)=\int_{0}^{u} \psi_{\delta}(v) d v, L_{\delta} \in C^{2}(\mathbb{R})$. Let $\gamma>0$. Multiplying the equation in (2) above by $\left(t-t_{0}\right)^{\gamma} \phi_{\delta}^{\prime}(u)$ and integrating in $\mathbb{R} \times\left[t_{0}, t\right]$, we get

$$
\begin{aligned}
& \int_{t_{0}}^{t} \int_{\mathbb{R}}\left(\tau-t_{0}\right)^{\gamma} \phi_{\delta}^{\prime}(u(x, \tau)) u(x, \tau)_{\tau} d x d \tau+\int_{t_{0}}^{t} \int_{\mathbb{R}}\left(t-t_{0}\right)^{\gamma} \phi_{\delta}^{\prime}(u(x, \tau))(f(\tau, u))_{x} d x d \tau \\
= & \int_{t_{0}}^{t} \int_{\mathbb{R}}\left(\tau-t_{0}\right)^{\gamma} \phi_{\delta}^{\prime}(u(x, \tau))\left(|u|^{\alpha} u_{x}\right)_{x} d x d \tau
\end{aligned}
$$

By Fubini's theorem, integrating by parts, using an appropriate cut-off function and taking $\delta \rightarrow 0$, this gives

$$
\begin{aligned}
& \left(t-t_{0}\right)^{\gamma}\|u(x, t)\|_{L^{q}(\mathbb{R})}^{q}+q(q-1) \int_{t_{0}}^{t}\left(\tau-t_{0}\right)^{\gamma} \int_{\mathbb{R}}|u(x, \tau)|^{\alpha+q-2}\left(u_{x}\right)^{2} d x d \tau \\
\leq & \gamma \int_{t_{0}}^{t}\left(\tau-t_{0}\right)^{\gamma-1}\|u(x, \tau)\|_{L^{q}(\mathbb{R})}^{q} d \tau
\end{aligned}
$$

Introducing

$$
v^{[q]}(x, t):=\left\{\begin{array}{l}
u(x, t) \text { se } \sigma=\alpha+q=2, \\
|u(x, t)|^{\sigma / 2}, \sigma=\alpha+q>2,
\end{array}\right.
$$

we then have

$$
\begin{aligned}
& \left(t-t_{0}\right)^{\gamma}\left\|v^{[q]}(\cdot, t)\right\|_{L^{2 q / \sigma}(\mathbb{R})}^{2 q / \sigma}+\frac{4 q(q-1)}{(\alpha+q)^{2}} \int_{t_{0}}^{t}\left(\tau-t_{0}\right)^{\gamma}\left\|v_{x}^{|q|}(\cdot, \tau)\right\|_{L^{2}(\mathbb{R})}^{2} d \tau \\
\leq & \gamma \int_{t_{0}}^{t}\left(\tau-t_{0}\right)^{\gamma-1}\left\|v^{[q]}(\cdot, \tau)\right\|_{L^{2 q / \sigma}(\mathbb{R})}^{2 q / \sigma} d \tau
\end{aligned}
$$


Using Hölder, Nirenberg-Sobolev-Gagliardo II (5), with $\beta_{0}=2 q / \sigma$ and $q=2 p_{0}$, and Nirenberg-Sobolev-Gagliardo I (4) inequalities, we obtain the supnorm estimate (6).

Let $w(\cdot, t)$ be the solution of (2) with initial condition $w_{0}=u_{0}^{+}+\epsilon \zeta$ for some $\epsilon>0$, where $u_{0}^{+}$denotes the positive part of $u_{0}$ and $\zeta \in C^{0}(\mathbb{R}) \cap L^{p_{0}}(\mathbb{R}) \cap L^{\infty}(\mathbb{R})$. That is, $w_{0} \geq u_{0}$. Then, by the Theorem of Comparison $(2.1), u(\cdot, t) \leq w(\cdot, t)$, for all $0 \leq t<T$ and

$$
\|w(\cdot, t)\|_{L^{\infty}(\mathbb{R})} \leq K\left(\alpha, p_{0}\right)\left\|w\left(\cdot, t_{0}\right)\right\|_{L^{p_{0}(\mathbb{R})}}^{\frac{2 p_{0}}{\alpha+2 p_{0}}}\left(t-t_{0}\right)^{-\frac{1}{\alpha+2 p_{0}}}, \quad \forall t, 0 \leq t_{0}<t,
$$

Now let $z(\cdot, t)$ be the solution of $(2)$ with initial condition $z_{0}=-u_{0}^{-}-\epsilon \zeta$ for some $\epsilon>0$, where $u_{0}^{-}$denotes the negative part of $u_{0}$. That is, $z_{0} \leq u_{0}$. Then, by the Theorem of Comparison $(2.1), u(\cdot, t) \geq w(\cdot, t)$, for all $t, 0 \leq t<T$ and

$$
\|z(\cdot, t)\|_{L^{\infty}(\mathbb{R})} \leq K\left(\alpha, p_{0}\right)\left\|z\left(\cdot, t_{0}\right)\right\|_{L^{p_{0}}(\mathbb{R})}^{\frac{2 p_{0}}{\alpha+2 p_{0}}}\left(t-t_{0}\right)^{-\frac{1}{\alpha+2 p_{0}}}, \quad \forall t, 0 \leq t_{0}<t,
$$

By (7) and (8), we have

$$
\|u(\cdot, t)\|_{L^{\infty}(\mathbb{R})} \leq K\left(\alpha, p_{0}\right) \max \left\{\left\|u_{0}^{+}\right\|,\left\|u_{0}^{-}\right\|\right\}_{L^{p_{0}(\mathbb{R})}}^{\frac{2 p_{0}}{\alpha+2 p_{0}}}\left(t-t_{0}\right)^{-\frac{1}{\alpha+2 p_{0}}}, \quad \forall t, 0 \leq t_{0}<t .
$$

This proves the following theorem:

Theorem 3.2. If $u(\cdot, t) \in L_{\text {loc }}^{\infty}\left(\mathbb{R}, L^{\infty}(\mathbb{R})\right)$ solves problem (2), then

$$
\|u(\cdot, t)\|_{L^{\infty}(\mathbb{R})} \leq K\left(\alpha, p_{0}\right) \max \left\{\left\|u_{0}^{+}\right\|,\left\|u_{0}^{-}\right\|\right\}_{L^{p_{0}(\mathbb{R})}}^{\frac{2 p_{0}}{\alpha+2 p_{0}}}\left(t-t_{0}\right)^{-\frac{1}{\alpha+2 p_{0}}}, \quad \forall t, 0 \leq t_{0}<t,
$$

where $K\left(\alpha, p_{0}\right)$ is a constant that only depends on $\alpha$ and $p_{0}$ and $u_{0}^{+}$and $u_{0}^{-}$denote the positive and negative part of $u_{0}$, respectively.

\section{Conclusions}

We derived a supnorm estimate for the solution of the porous medium equation (2) with no restriction on the sign of $u_{0}$.

\section{Acknowledgments}

We thank Prof. Paulo Zingano for comments and fruitful discussions. 


\section{References}

[1] E. DiBenedetto. Partial Differential Equations. Birkhauser, Boston, 2000.

[2] L. Fabris. Sobre a existência global e limitação uniforme de soluções da equação dos meios porosos com termos advectivos arbitrários. Tese de Doutorado, PPGMAT/UFRGS, 2013.

[3] L. Schütz, J. S. Ziebell, J. P. Zingano and P. P. Zingano. A new proof of a fundamental supnorm estimate for one-dimensional advection-diffusion equations. Advances in Differential Equations and Control Processes, Vol. 11, Number 1, pages 41-51. Pushpa Publishing House, India, 2013.

[4] J. M. Urbano. The Method of Intrinsic Scaling. Springer, Berlin, 2000.

[5] J. L. Vázquez. The Porous Medium Equation - Mathematical Theory. Oxford Mathematical Monographs. Oxford Science Publication, New York, 2007. 